\title{
Les travaux paravalanches de Saint Colomban des Villards
}

\author{
PAR
}

\section{Anchierri}

lugénienr en Chef des Fuux ef Forêts

Les travaux de protection contre les avalanches sont it l'ordre du jour. Ainsi, les travaux realisés à Saint Colomban des Villards commencés maintenant depuis plus de vingt ans connaissent-ils aujourd'hui une certaine notoriété, d'ailleurs justifiée par l'exemple d'une réalisation cohí rente qu'ils représentent.

\section{Saint Colomban des Villards et ses avalanches.}

La Commune de Saint Colomban des Villards occupe tout le haut de la Vallée du Glandon, un affluent de rive gauche de l'Arc en moyenne Maurienne.

C'était jadis une commune extrêmement peuplée, puisqu'elle a compté en 18801350 habitants, et qui fournissait, à la mauvaise saison, un très important contingent des célèbres ramoneurs savoyards et aussi do colporteurs.

Son déclin s'est amorcé, comme pour la quasi totalité des Communes de montagne, au début de ce siècle. La guerre de 1914-1918 - à laquelle elle a payé un tribut terriblement lourd de 67 tués et combien de blessés Ia accéléré et, actuellement, elle cherche désespérement à survivre en s'appuyant notamment sur le tourisme.

Les "villards" (hameaux caractéristiques très groupés qui ont donné leur nom à la vallée) de Saint Colomban sont établis sur les deux rives du Glandon, lequel, très schématiquement, coule au contact des deux formations géologiques essentielles que l'on rencontre dans la vallée : à l'Ouest-Nord Ouest, la haute chaine des massifs cristallins externes, à l'Est-Sud Est les assises liasiques à fort pendage vers l'Est de la couverture sédimentaire des massifs cristallins ("zone dauphinoise"). La rivière s'est profondément enfoncée dans les assises schisteuses ou schisto-calcaires du Lias et le profil en travers en $\mathrm{V}$ de la vallée est très accentué : les avalanches de neige font depuis toujours partie de l'environnement et les villards. justement, se sont édifiés autant que faire se peut sur les petits replats existants abrités - ou censés l'être - de ces avalanches.

Aucune des deux rives du Glandon n'est à l'abri, mais la rive gauche, située à l'Adret et plus accueillante, se trouve être tout à la fois la plus peuplée - elle compte 4 villards, contre 3 en fort déclin sur la rive droite et la plus exposée en raison du relief plus tourmenté et de l'altitude plus grande de la chaine cristalline qui culmine vers 2800 mètres au droit de Saint Colomban.

Et comme bien d'autres communes de montagne des Alpes, Saint Colomban des Villards, malgré une population nous le rappelons très nombreuse, s'était accommodée de cette situation : les villages, en grande partie désertés I'hiver par les hommes valides, vivaient repliés sur euxmêmes et, en évitant soigneusement les couloirs dangereux pendant les périodes avalancheuses, les victimes demeuraient exceptionnelles.

Mais la vallée des Villards est l'une des plus verdoyantes et des plus belles de Savoie. Comme, au surplus, une bonne route desservait la vallée et la reliait par le Col du Glandon et le Col de la Croix de fer aux vallées de l'Eau d'Olle et de la Romanche et au bassin de l'Arvan, le tourisme s'y est développé très vite : deux hôtels de tourisme ont été édifiés au chef-lieu entre 1918 et 1939 et Saint Colomban figurait sur les cartes des stations de sports d'hiver éditées avant 1939 par la Cie P.L.M.

En 1936 et surtout en 1942 des avalanches détruisent au chef-lieu une partie de l'un des deux hôtels et, au vil- 
lage du Martinan, deux immeubles, les uns et les autres sans doute édifiés un peu inconsidérément en bordure des couloirs dangereux. Il y a 6 morts et l'avalanche. avec laquelle on vit depuis des siècles, apparait soudain un fléau insupportable : Saint Colomban des. Villards fait appel à la collectivité nationale pour hi venir en aide.

1942 : rares, très rares, sont en France les personnes compétentes en matière d'avalanches : mis à part peutêtre les pieux en fonte implantés par le Génie militaire pour protéger Barèges, on en est demeuré pour l'essentiel à la technique des murs et banquettes. Au surplus la période de guerre n'est pas propice à l'exécution de travaux.

En revanche, le principe de la prise en charge des travaux de protection des divers villages et de la route $\mathrm{Na}$ tionale 527 du Col du Glandon semble assez facilement admis, la Commune acceptant de céder gratuitement à l'Etat une importante surface de terrains communaux. en partie boisés, dans la zone de départ des avalanches.

\section{Le problème posé et les solutions adoptées.}

L'Etat, par l'Administration des Eaux et Forêts, a dono admis de prendre intégralement a sa charge la protection contre les avalanches des villages de Saint Colomban, ceux du moins qui sont situés sur la rive gauche du Glandon.

C'est assurément une très lourde charge, sans doute injustifiée si, aujourd'hui, on ne prend en considération que les intérêts protégés.

Mais c'est aussi pour l'Administration des Eaux et Forêts, désormais maîtresse des travaux, l'occasion de concevoir un plan rationnel et complet pour la réalisation duquel elle va disposer du temps nécessaire, et aussi de crédits importants. Sans avoir à se soumettre aux impatiences et aux exigences souvent contradictoires d'élus locaux.

Et cette circonstance est d'autant plus intéressante que la période d'après-guerre - grâce surtout, à l'origine, aux chantiers d'E.D.F. implantés en altitude dans des sites parfois dangereux et qu'il faut coûte que coùte protéger car on veut travailler en hiver tant sont grands les besoins en énergie - voit se développer un brusque intérêt pour toutes les techniques de défense contre les avalanches.

Outre les méthodes de déclenchement artificiel, encore très incertaines, ce sont essentiellement les ouvrages actifs de rétention de la neige qui ont la vedette.

Les terrains domaniaux de Saint Colomban des Villards vont ainsi devenir tout à la fois un excellent banc d'essai des divers types d'ouvrages paravalanches et un exemple assez unique dans les Alpes (avec les travaux très analogues réalisés dans la Commune assez voisine de Celliers, en Tarentaise) d'un dispositif réfléchi et complet de travaux.

Parant au plus pressé, l'Administration, avant toute autre chose, édifie aux débouchés des couloirs dangereux des digues de déviation, sept au total. Ce sont des ouvrages massifs en maconnerie sèche de gros blocs épaulés avec de forts remblais ou bien en gabions métalliques.
Dans le couloir le plus critique, celui de la Combe Barral qui menace le Chef-Lieu, les ouvrages sont complétés par un reprofilage du couloir, au droit des deux digues, ce qui améliore considérablement leur efficacité. Il n'est sans doute pas exagéré de dire qu'une fois au moins depuis leur édification les digues de Barral ont évité une catastrophe.

Il est désormais possible de réfléchir posément aux solutions susceptibles d'apporter aux Villarins une protection satisfaisante, ce que, bien évidemment, n'apportent pas les digues de déviation.

Des solutions qui, bien entendu, vont constituer un compromis entre le souhaitable et le possible.

Première opération : parmi les avalanches qui dévalent le versant, distinguer celles susceptibles d'être éteintes dans les zones de départ et celles dont l'extinction exigerait des travaux titanesques.

Pour ce choix, une considération importante : les ouvrages "actifs" sont particulièrement onéreux à édifier et aussi à entretenir. Il convient donc de prendre largement en compte la possibilité de faire relayer les ouvrages par la Forêt, ce qui implique immédiatement la fixation d'une limite altitudinale que l'on peut, dans la vallée des Villards, à l'exposition S.E., fixer, assez généreusement, à $2000 \mathrm{~m}$

Les deux considérations qui précèdent - volume d'ouvrages humainement réalisable compte-tenu du but recherché et ouvrages devant en principe être situés dans la zone de végétation forestière - conduisent à renoncer à la correction des pentes alimentant, au Sud des zones menacées, le couloir de Combe Barral et, au Nord de celles-ci, celui des Trois Croix.

En rewanche, par une circonstance exceptionnellement favorable, les avalanches descendant entre le Chef-lieu et le Martinan - les deux principaux villages - qui menacent littéralement de couper en deux la Commune prennent naissance entre $1500 \mathrm{~m}$ et $2000 \mathrm{~m}$ et donc entrent dans les limites fixées pour l'exécution d'ouvrages actifs.

Si l'on considere au surplus que les avalanches descendant les "3 Croix" n'atteignent pratiquement jamais la route nationale, la stabilisation du versant entre le Martinan, la Cure et le Chef-Lieu va donc apporter à Saint Colomban des Villards un atout considérable pour son développement touristique hivernal.

Le plan général des travaux paravalanches de Saint Colomban des Villards, est désormais tracé :

- Consolider et améliorer les digues de déviation édifiées aux débouchés des couloirs des 3 Croix et surtout de Barral (les digues de Barral dévient l'avalanche au SudOuest du Chef-Lieu et protègent donc la zone privilégiée). Délaisser au contraire les autres digues de déviation (La Cure, Le Martinan).

- Edifier sur le versant de Frémezan, jusqu'à $2000 \mathrm{~m}$ environ, un réseau complet d'ouvrages de rétention de la neige.

- Boiser tous les terrains aussitôt après la construction des ouvrages.

Il s'agit là du schéma général mais, bien entendu, les travaux ont été exécutés en cherchant à profiter au maximum du terrain et à réduire simultanément autant que 
faire se pouvait, les zones d'alimentation des couloirs des 3 Croix et de Barral à l'aide de travaux légers et surtout de boisements.

\section{Les réalisations.}

Sur le terrain, la mise en ouvre du programme exposé ci-dessus a conduit aux réalisations ci-après :

\section{a) La route forestière de Frémezan}

La pièce maitresse de la correction est constituée, nous l'avons dit, par les ouvrages de rétention de la neige à édifier sur le versant de "Frémezan" entre $1500 \mathrm{~m}$ et $2000 \mathrm{~m}$ environ.

En première approximation, compte-tenu de la technique de 1950, il apparait que le tonnage de matériaux nécessaire pour 1 mètre lináire de rateliers ou de claie est de l'ordre de $1 \mathrm{~T}$ (y compris les ancrages en béton). Le plan général de correction prévoyant l'édification d'environ 2000 mètres linéaires de rateliers, c'est un tonnage de 2000 T qu'il faut transporter de la Route Nationale à $1100 \mathrm{~m}$ jusqu'à une altitude moyenne de $1700 / 1800 \mathrm{~m}$ : il y faut soit un téléphérique, soit une route.

La solution route est retenue en raison de sa souplesse d'utilisation, des grandes facilités qu'elle apporte pour les transports de main-d'ceuvre, de sa pérennité - moyennant entretien - qui sera très précieuse pour l'entretien des ouvrages et des boisements, enfin, considération importante, de son rôle actif comme ouvrage paravalanches sur environ $4 \mathrm{kms}$.

C'est ainsi une route d'environ $8 \mathrm{kms}$ qui est édifiée dans le versant avalancheux avec une plateforme de $3 \mathrm{~m} 50$ à $4 \mathrm{mo0}$ et un volume important de maçonneries : des murs de soutènement qui, renforcés avec des I.P.N. et des grillages, constituent d'excellents murs paravalanches.

Régulièrement entretenue et aujourd'hui revêtue, la "route de Frémezan" a supporté un trafic de poids lourds important ... sans compter les transports quotidiens d'ouvriers, les voitures de tourisme et même les autocars!

\section{b) Les ouvrages charpentés}

Je ne m'étendrai pas sur les divers types d'ouvrages charpentés édifiés à Frémezan. Aujourd'hui chacun connait peu ou prou ces ouvrages qui ont noms claies a neige (autrefois pont à neige) et rateliers à neige. Nous avons édifié à Saint Colomban des Villards à peu près tous les types d'ouvrages, du "tout bois" jusqu'au "tout acier", de la claie à la palissade et aux rateliers, l'ouvrage de loin le plus construit en raison de son prix de revient relativement modique étant le ratelier à neige en rondins résineux injectés à la créosote reposant sur des socles d'ancrage en béton.

Une caractéristique commune à tous ces ouvrages : des ancrages très généreusement calculés (socles amont de $1 \mathrm{~m}^{3}$ et parfois plus) grâce auxquels les ouvrages de Saint Colomban ont pu "tenir le coup" au cours de nombreux hivers critiques.

\section{c) Les autres ouvrages de rétention}

L'ouvrage charpenté demeure un ouvrage onéreux et, chaque fois que les conditions du terrain - pente, état de sol, etc - ou un risque moindre en cas de déclenchement (combes secondaires se déversant dans les grands couloirs de Barral ou des 3 Croix) l'ont permis, nous avons substitué au ratelier des banquettes terrassées généralement consolidées avec des rails et grillages, ou des barrières.

Les murs de soutènement de la route forestière ont cux aussi, je le rappelle, leur róle et la route elle-méme constitue une banquette d'une exceptionnelle largeur.

Signalons enfin que "lexploitation du terrain" nous a conduit à édifier, au pied d'un couloir difficile et sans doute très contteux à stabiliser, deux "poches" d'accumulation analogues à ce que nous avons pu voir à Parthenen dans le Montafon ou à l'Hafelekar à Innsbruck. Ces poches ont été jusqu'ici capables d'accumuler les coulées détachées au-dessus.

\section{d) Les boisements}

Ils ont été entrepris dès 1953 dans tous les terrains cédés à l'Etat et sur lesquels n'était pas prévue l'édificalion d'ouvrages. Ils ont ensuite été poursuivis systématiquement jusque vers $2000 \mathrm{~m}$ au fur et à mesure de l'édification des divers ouvrages, les essences utilisées étant essentiellement l'épicéa et le mélèze, ce dernier surtout en altitude.

Jusqu'en 1970, ces boisements étaient, à juste titre, l'orgueil du Service R.T.M. Savoyard.

Les années d'enneigements absolument extraordinaires que nous avons connues depuis 1970 ont malheureusement porté de rudes coups aux plantations situées au-dessus de $1750 \mathrm{~m}-1800 \mathrm{~m}$ selon l'orientation : le mélèze surtout, malgré sa réputation de "chêne de la montagne" a payé un très lourd tribut et nous avons vu disparaitre en 3 ou 4 ans des hectares de plantations hautes déjà de $1 \mathrm{~m} 50$ à $2 \mathrm{mo0}$.

Ce grave accident nous a obligé à reconstituer les peuplements détruits en faisant appel aux Pins de préférence au mélèze : pin à crochet et Pin Cembro (Arolle) mais, de toute façon, quinze années, parfois vingt, ont été perdues.

\section{Les résultats.}

Depuis l'édification du réseau paravalanches de Frémezan, Saint Colomban des Villards a connu plusieurs hivers très neigeux et dont certains ont été ailleurs catastrophiques.

Le phénomène avalancheux est cependant trop fantasque et trop localisé pour qu'il soit permis de proclamer que Saint Colomban est désormais à l'abri des avalanches.

Il reste cependant ceci :

En 1961, de très violentes avalanches ont dévalé les couloirs des 3 Croix et, surtout, de Barral. Les deux digues de déviation de Barral ont été près d'être submergées, 
mais elles ont parfaitement détourné les avalanches dont l'une a atteint de Glandon.

- Il est donc permis de dire que cette année-là les conditions étaient propices, à Saint Colomban, au déclenchement des avalanches. Or, aucune des avalanches recensées de "la Cure" et du "Martinan" qui prenaient naissance dans le versant de Frémezan ne s'est manifestée, attestant de l'efficacité du dispositif mis en place.

- Depuis 1961, les tournes de Barral ont été considérablement renforcées et surélevées. De nouveaux rateliers ont été édifiés là où quelques petits départs ont pu être repérés. L'ensemble est régulièrement entretenu.

- Et pendant ce temps, la forét, jusque vers $1700 / 1800 \mathrm{~m}$. se constitue "ensevelissant" par place certains ouvrages. Au-dessus, nous espérons que les nouveaux boisements de 1973-74 et 75 auront un meilleur sort que les précédents.

Techniquement on peut donc dire que les travaux paravalanches de Saint Colomban des Villards sont une belle réussite. Une réussite qui pourrait servir d'exemple et qui souligne en tous cas le fait que là non plus le succès ne s'improvise pas.

\section{Quelques chiffres.}

Route d'accès : longueur $7 \mathrm{~km} 800$ - bitumée. plus de $4000 \mathrm{~m}^{3}$ de murs maçonnés.

"Tournes" paravalanches : 7. Environ $8500 \mathrm{~m}^{3}$ de maçonnerie sèche, $1950 \mathrm{~m}^{3}$ de gabions, $5000 \mathrm{~m}^{3}$ de ter rassements.

Rateliers, claies et palissades : $2110 \mathrm{~m}$.

Banquettes: $2841 \mathrm{~m}$; dont $1191 \mathrm{~m}$ de banquettes de $2 \mathrm{~m} 50$ de largeur renforcées avec rails et grillages.

Boisements : 62 ha effectivernent boisés en épicéas, mélèzes (reconstitution en pins sur 5 ha).

Valeur 1974 de l'ensemble des travaux : 5550000 Francs (y compris l'entretien depuis les débuts).

\section{Discussion}

Président : M.L. de CRECY

M. le Président remercie M. ANCHIERRI d'avoir parfaitement décrit et illustré des travaux qui constituent probablement l'expérience la plus complète réalisée en France dans ce domaine. ll ouvre ensuite la discussion.

Sur une question de M. ROUILLON, M. ANCHYERRl apporte les informations, ci-après :

Les terrains sur lesquels ont été implantés les ouvrages étaient surtout des terrains communaux qui ont été cédés à l'Etat à des prix "assez symboliques" ; mais nous avons dú acquérir certains terrains privés.

C'est la municipalité qui a réclamé l'exécution des travaux paravalanches ; la population était fort sceptique et semblait estimer "que l'on n'empêcherait jamais les avalanches de descendre". Aujourd'hui, elle est convaincue de l'efficacité des travaux mais est portée à penser qu'ils ont coûté trop chers ... bien qu'elle n'en ait pas supporté les frais. Aujourd'hui, la Commune réclame d'autres ouvrages.

M. BF.ZINGE: souhaiterait quelques renseignements complémentaires sur les peuplements spontanés de pins arolle et de mélèzes sur le versant Sud de Saint-Colomban.

\section{PONCET répond comme suit :}

Nous sommes certainement dans l'aire d'extension du Pin Cembro (arolle) qui a probablement été chassé par les pratiques pastorales du pays.

La question de la spontanéité du mélèze est plus discutée . . La découverte d'un très vieux mélèze vivant très haut sur l'autre versant de la montagne (N.O.) plus éloigné du centre de gravité de l'aire de l'espèce, donne à penser que nous sommes encore dans l'aire géographique de cette dernière qui est devenue rare par la concurrence d'autres essences forestières plus sociales et mieux adaptées à un climat assez humide.

11 poursuit en posant à M. ANCHIERRI la question suivante :
A-t-on constaté, à la suite des gels précoces de Septembre 1974 sur mélèzes encore verts, du "faux printemps" de Noël à la miMars 1975 et des gels tardifs d'Avril-Mai, d'importants dégâts de gel sur les jeunes pousses des mélèzes?

M. ANCHIERRI répond que ces dégâts se sont confondus avec ceux causés préalablement par les attaques de pucerons et en ont sans doute aggravé les dégâts.

Quelle était la provenance des plants de mélèzes utilisés? de mande M. BAZIRE.

M. ANCHERRI the peut préciser s'il s'agit de graines fournies il y a longtemps par l'Administration ou de plants achetés à un pépiniériste privé.

Je crois que pour ces derniers, précise M. PONCET, l'origine des graines était sans doute autrichienne ou orientale, provenances de basse altitude, à végétation vigoureuse s'étendant sur une longue saison (débourrage précoce, défoliaison tardive) recherchées pour le reboisement de production en basses montagnes comme celles des Sudètes ou de Pologne),

Or, même au Tyrol, les boisements d'altitude réalisés depuis une vingtaine d'années avec une telle provenance de mélèzes (d'altitude modérée) ont énormément souffert de brûlures des rameaux et jeunes cimes par les gels hors saison de Septembre 1974 -- AvrilMai 1975 avec "faux printemps" intercalé.

Les dégâts de gel constatables sur les semis naturels et les rameaux des mélezins d'altitude du haut Dauphiné (Briançonnais, Queyras) sont beaucoup moins graves et étendus.

Il est probable que, pour nos boisements de protection en altitude, près de la limite supérieure de la végétation forestière, il faille revenir à l'utilisation de races ou provenances adaptées à l'altitude, à croissance lente, comme celles de nos vallées intraalpines françaises, où il: convient d'organiser, comme autrefois, des récoltes de graines pour nos pépinières. 


\begin{tabular}{|c|c|}
\hline \multicolumn{2}{|c|}{$\begin{array}{l}\text { Abstract } \\
\text { Avalanche protection for Saint Colomban des Villards in the French Alps }\end{array}$} \\
\hline $\begin{array}{l}\text { The avalanche protection scheme for the Saint Colomban des } \\
\text { Villards district, which is to ensure the safety of several villages, } \\
\text { is unique in France by the complete, efficient protection it is designed } \\
\text { to provide. } \\
\text { Owing to its high cost and long-term implementation schedule, } \\
\text { the project was taken over by the French Government, which } \\
\text { ensured that the Forestry Authorities would have the time and } \\
\text { facilities they required for preparing a rational work schedule and } \\
\text { putting it into effect over a period of } 20 \text { years. } \\
\text { As the first and most urgent requirement, several "passive" } \\
\text { protection works were installed (diversion dykes at the outlets } \\
\text { from the main avalanche corridors). } \\
\text { An access road was then built to areas it was considered could } \\
\text { be stabilized at reasonable cost. } \\
\text { Next, various conventional types of snow barrier were installed, } \\
\text { ranging from simple banks to racks capable of withstanding the }\end{array}$ & $\begin{array}{l}\text { heaviest snow loads. } \\
\text { Finally, trees were planted between, in and around the protection } \\
\text { works. Despite some failures caused by a series of exceptionally } \\
\text { snowy winters, retimbering seems to have been successful on the } \\
\text { whole; the new trees at various sites should be capable of taking } \\
\text { over from the anti-avalanche works within the next } 10 \text { to } 25 \text { years. } \\
\text { Even taking a very conservative view of the results so far, it } \\
\text { seems reasonable to claim that the anti-avalanche works in the } \\
\text { Saint Colomban des Villards district have been effective during } \\
\text { several very snowy winters, in which unprotected areas suffered } \\
\text { disastrous avalanches. } \\
\text { The project, which also includes the smaller Cellier district, is } \\
\text { the first of its kind to have been put into effect in the French Alps } \\
\text { in addition, it has provided the Forestry Authorities with excellent } \\
\text { scope for experimentation. The anti-avalanche works attract a good } \\
\text { many visitors to the area. }\end{array}$ \\
\hline
\end{tabular}

